\section{Electrocautery-induced pacemaker malfunction during surgery}

Devanand Mangar MD, ${ }^{*}$ Glen M. Atlas MD, $\dagger$

Peter B. Kane MD $\dagger$
We report a case of electrocautery-induced pacemaker failure that resulted in asystole in a 15 -year-old girl scheduled for cardiac surgery. Her pacemaker was converted to the asynchronous mode the night before surgery. Electromagnetic interference from the unipolar electrocautery caused a reducion in the battery voltage, which allowed the digital circuitry, but not the voltage control oscillator (VCO), to work properly. Eventually the battery current drain caused VCO "lock-out," and pacemaker and battery failure. This report demonstrates that electrocautery-induced pacemaker failure can occur, even after conversion to asynchronous mode.

Nous rapportons le cas du mauvais fonctionnement d'un pacemaker causé par l'électrocautère et qui a mené à une asystolie chez une patiente de 15 ans qui devait subir une chirurgie cardiaque. Son pacemaker a été converti à un mode asynchrone la nuit avant la chirurgie. L'interférence électromagnétique de l'électrocautère unipolaire a causé une diminution du voltage de la pile. Ceci a permis au circuit digital, mais non a l'oscillateur de contrôle du voltage (VCO), de bien fonctionner. Eventuellement, la fuite de courant de la pile a causé une mise en veilleuse du VCO, et une panne du pacemaker et de la pile. Ce communiqué nous démontre qu'une panne du pacemaker causée par l'électrocautère peut survenir, même après une conversion à un mode asynchrone.

\section{Key words}

COMPLICATIONS: arrhythmia, arrest, cardiac;

EQUIPMENT: cautery, pacemakers;

HEART: pacemaker, artificial.

From the Department of Anesthesiology, University of South Florida College of Medicine, ${ }^{*}$ Tampa, Florida and SUNY Health Science Center, $†$ Syracuse, New York.

Address correspondence to: Dr. Devanand Mangar, University of South Florida College of Medicine, Department of Anesthesiology, MDC Box 59, Tampa, Florida 33612-4799 Accepted for publication 6th March, 1991
Pacemakers are used to maintain the heart rate when the inherent conduction system of the heart fails, either because of disease or surgery. Most permanent pacemakers are programmable and lead to an increase in complexity and potential problems for anaesthetists during surgery. Some demand pacemakers will convert automatically to the asynchronous mode in the presence of intermittent or modulating electromagnetic fields. Multi-programmable pacemakers are now widely used and can be changed to the asynchronous mode before the patient comes to the operating room (OR). ${ }^{1-3}$ The asynchronous mode is beneficial during surgery because a unipolar electrocautery may interfere with pacemaker function. Although the generator remains in the voo (ventricular asynchronous type without sensing capability) mode, it may inadvertently be reprogrammed to some other mode by the electrocautery, causing irreversible damage to the pacemaker pulse generator. Electrocautery may increase the rate of capture of the pacemaker and also induce ventricular fibrillation. ${ }^{4}$ Electromagnetic interference (EMI) is defined as an adverse change in the normal function of a device caused by electromagnetic energy, either radiated or conducted. Such EMI has previously been shown to inhibit demand pacemakers. ${ }^{5-10}$

We observed pacemaker failure resulting in asystole caused by voltage control oscillator (VCO) "lock-out" in a patient whose pacemaker had been converted to the asynchronous mode the night before surgery.

\section{Case report}

A 15-year-old, $66 \mathrm{~kg}, 165 \mathrm{~cm}$ white girl presented with a history of left transposition of the great arteries, pulmonary atresia, and ventricular septal defect. Previous operations included Blalock-Taussig shunt, Waterston shunt, and a Rastelli procedure (LV-PA conduit). Following her last operation six years ago, she had third degree AV block that was managed by insertion of a ventricular pacemaker that sensed $\mathbf{R}$ waves to inhibit artificial pacing (vvi). On admission, the patient had moderate to severe conduit (LV-PA) and conduit valve stenosis, which required correction. The night before surgery the vvi pacemaker was changed to the asynchronous mode, because the unipolar electrocautery might interfere with the pacemaker function. 
After premedication with $5 \mathrm{mg}$ of diazepam po, the patient was brought to the $\mathrm{OR}$, and anaesthesia was induced with thiopentone $200 \mathrm{mg} i v$. The trachea was intubated with a $7.0 \mathrm{~mm}$ cuffed tube following administration of $1 \mathrm{mg}$ of pancuronium, and $80 \mathrm{mg}$ of succinylcholine iv. A 20-ga right radial artery catheter and left triple lumen subclavian catheter were inserted. Electrocautery, used to control bleeding, caused transient asystole, which reverted to normal with cessation of cauterization. This phenomenon recurred with the second use of the electrocautery. On the third use, the patient remained asystolic, despite cessation of cauterization. Cardiopulmonary resuscitation was started, generating a systolic blood pressure of $80 \mathrm{mmHg}$. Atropine $0.4 \mathrm{mg}$ and isoproterenol $0.1 \mu \mathrm{g} \cdot \mathrm{kg}^{-1}$ were administered through the triple lumen catheter. Four minutes later, the heart rate was 40 beats per minute (bpm), and systolic blood pressure was $80 \mathrm{mmHg}$. In order to isolate the pacemaker wires from the pulse generator, the surgeon opened the area over the generator, which was located in the left side of the abdomen. The wires were connected to an external pacemaker and the heart was paced at $60 \mathrm{bpm}$. The operation then proceeded uneventfully. Before weaning the patient from cardiopulmonary bypass, a new pacemaker generator was inserted. The patient was taken to the ICU and was discharged from the hospital several days later. The pacemaker generator was returned to the manufacturer for analysis.

\section{Discussion}

In this case, meticulous attention was given to ensure that pacemaker failure would not occur. Precautions included reprogramming the pacemaker to the asynchronous mode in addition to proper placement of the grounding pad. In spite of our efforts, pacemaker malfunction occurred. A pulse generator has two major components: a lithium battery and electronic circuitry. The electronics consist of a voltage control oscillator (VCO) and digital circuitry (Figure). The VCO emits a signal to the digital circuitry during a pacing pulse. The number and timing of the signals emitted from the VCO determine the duration of the pulse and are dependent upon the programming of the generator's pulse width (duration). For example, if the pulse width is programmed to $0.5 \mathrm{msec}$, the VCO must emit 20 signals to the digital circuitry before the digital circuitry will shut off the VCO and terminate the output pulse.

When the VCO is functioning, it produces current drain from the battery. When the battery voltage is adequate, the VCO transmits the appropriate signals to the digital circuitry, which then shuts off the VCO and thereby reduces further battery drain. Battery voltage must be at least 1 to 1.5 volts for the VCO to send the proper signals.

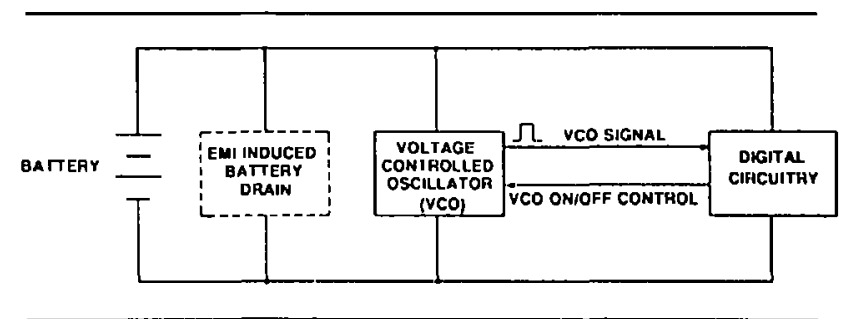

FIGURE Electronic circuit of a pacemaker generator showing voltage control oscillator (VCO), digital circuitry, and battery with electromagnetic-induced (EMI) battery drain.

If battery voltage decreases below 1 to 1.5 volts, the signals emitted from the VCO will be unable to activate the digital circuitry. The digital circuitry will "request" an output pulse, timed according to the programmed rate, from the VCO, and the VCO will remain on. The digital circuitry continues to wait for the correct number of signals so it can shut off the VCO. Since it does not receive the signals, the $\mathrm{VCO}$ circuit keeps running and creates additional current drain that results in even lower battery voltage. This condition is referred to as VCO "lock-out."

Cold temperatures and electrocautery are exogenous conditions that can decrease the battery voltage until VCO "lock-out" can occur. The exact effect of the electrocautery on a pacemaker pulse generator is unpredictable. Often, it will turn on circuits and transistors that would normally be off and result in current drain that can reduce battery voltage below the level required for proper VCO function. Clinically, the effect is seen as an apparent loss of pacemaker output.

When the pacer was analyzed by the manufacturer (Medtronic), the filtered voltage of the generator was 1.011 volts, a voltage level consistent with exposure to electrocautery. With the exception of the battery, all other components of the pulse generator were within specifications. When a new 2.8 volt battery was connected to the circuitry, the pacemaker functioned normally. The voltage at which VCO "lock-out" can occur spontaneously is well below the end-of-service (battery depletion) level of the 2.1 to 2.3 volts recommended by the manufacturer.

When unipolar electrocautery is to be used during an operation, the grounding plate should be placed as close to the operative site and as far from the pacemaker as possible. Electrocautery should not be used within $15 \mathrm{~cm}$ of a pacemaker pulse generator, and its use should be limited to one-second bursts every ten seconds to prevent repetitive asystolic periods. If a demand pacemaker is inhibited by electrocautery, a high-powered magnet should be placed over the generator to convert it to the asynchronous mode. The use of bipolar electrocautery forceps further reduces the chance of electromagnetic 
interference, ${ }^{10,11}$ since a grounding plate is not used and the current flows between the two tips of the forceps. In addition, a transcutaneous pacemaker, isoproterenol, and atropine should be available to treat unexpected sudden cessation of pacing.

We believe that electromagnetic interference from electrocautery caused reduced battery voltage. This enabled the digital circuitry, but not the VCO, to work properly. The VCO was not turned off by the digital circuit, because appropriate signals were not received. Eventually, battery current drain was sufficient to cause VCO "lock-out" and pacemaker and battery failure. When unipolar electrocautery is anticipated in patients who have third degree AV blocks and permanent pacemakers, inserting a transvenous pacemaker the night before surgery should be considered. As shown by this case, pacemaker failure can occur despite conversion to the asynchronous mode.

\section{References}

1 Zaidan JR. Pacemakers. Anesthesiology 1984; 60: 319-34.

2 Parsonnet V, Furman S, Smyth NP. A revised code for pacemaker identification. Pace 1981; 4: 440-3.

3 Erdman S, Levinsky L, Servadio C, Stoupel E, Levy $M J$. Safety precautions in the management of patients with pacemakers when electrocautery operations are performed. Surg Gynecol Obstet 1988; 167: 311-4.

4 Levine PA, Balady GJ, Lazar HL, Belott PH, Roberts AJ. Electrocautery and pacemakers: management of the paced patient subject to electrocautery. Ann Thorac Surg 1986; 41: 313-7.

5 Scott $D L$. Cardiac pacemakers as an anaesthetic problem. Anaesthesia 1970; 25: 87-104.

6 Fleming WH, Toler JC. Degradation of pacemaker function by electromagnetic interference. Circulation (suppl III) 1974; 50: 226.

7 Batra $Y K$, Bali $I M$. Effect of coagulating and cutting current on a demand pacemaker during transurethral rescction of the prostate. A case report. Can Anaesth Soc J 1978; 25: 65-6.

8 Lerner SM. Suppression of a demand pacemaker by transurethral electrocautery. Anesth Analg 1973; 52: 703-6.

9 Smith RB, Wise WS. Pacemaker malfunction from urethral electrocautery (Letter to the Editor). JAMA 1971; 218: 256.

10 Wynands JE. Anesthesia for patients with heart block and artificial cardiac pacemakers. Anesth Analg 1976; 55: 626-32.

11 Simon $A B$. Perioperative management of the pacemaker patient. Anesthesiology 1977; 46: 127-31. 